\title{
Combined Use of DIC, EBSD and Simulation to Understand the Microscale Plastic Strain Distribution in Mg Alloys
}

\author{
Chad W. Sinclair ${ }^{1}$, Guilhem Martin ${ }^{2}$, Ricardo A. Lebensohn ${ }^{3}$ \\ ${ }^{1}$ Department of Materials Engineering, The University of British Columbia, Vancouver, BC, V6T 1 Z4 \\ CANADA \\ ${ }^{2}$ Laboratoire de Science et Ingénierie des Matériaux et Procédés, Groupe GPM2, Domaine \\ Universitaire, 101 rue de la physique 38402 Saint Martin d'Hères FRANCE \\ ${ }^{3}$ Materials Science and Technology Division, Los Alamos National Laboratory, Los Alamos, NM \\ 87544, USA
}

In response to government demands for reduced vehicle emissions, industry has targeted the reduction of vehicle weight as a key strategy. Reduced vehicle weight leads to reduced consumption and emissions, but also aids adoption of new propulsion technologies such as electric, hybrid and fuel cells. A key challenge in this is to identify materials that meet both structural and cost constraints.

One of the concerns in developing magnesium sheet with room temperature formability, is the complex deformation behavior it exhibits compared to other structural alloys. Owing to its strong plastic anisotropy, crystallographically informed models are required for the prediction of plasticity and failure. Significant effort has been made to develop and use crystal plasticity models to predict the macroscopic stress-strain response of magnesium alloys both under simple monotonic loading paths (e.g. tension and compression along different loading directions), e.g. [1], and more complex loading paths (e.g. various biaxial loading paths), e.g. [2]. Our work in this area has relied heavily on the use of mean-field selfconsistent crystal plasticity simulations (i.e. the Los Alamos Visoplastic Self Consistent Model [3]). Recognizing, however, the microscale plastic strain heterogeneity found in magnesium alloys, however, raises questions about the limits of such mean-field assumptions.

As a first attempt at measuring and interpreting the spatial distribution of plastic strain, experiments were designed to study two magnesium-rare earth alloys under slip-dominated deformation conditions [4-5]. A Mg-1Zn-0.5Nd (all in wt\%) alloy [4] and a commercial ZEK100 alloy[5] were prepared as sheet material and tested in tension parallel to the prior rolling direction. While the macroscopic stressstrain behavior was recorded, so too was the evolution of the local plastic strain. This was achieved by using e-beam lithography to deposit a gold grid on the surface of the sample (figure 1a). The grid size was selected so as to allow for a large area of investigation while ensuring a significant number of grid intersections per grain (grain size was 30-40 $\mu \mathrm{m}$ for each material). Digital image correlation was then used to measure local plastic strain on the surface of the sample progressively after different levels of macroscopically imposed plastic strain. At the same time, electron backscatter diffraction measurements were made in the same area as the grid so as to allow for correlation between local strain and crystallographic orientation. The results of these measurements showed that significant plastic strain heterogeneity existed within the materials and that this strain heterogeneity was not a form of strain localization. This was confirmed by the observation that the distribution of plastic strain was selfsimilar for all levels of measured plastic strain [5]. Second, it was found that no simple correlation existed between the starting crystallographic orientation of a point within the material and the level of plastic strain it accumulated. Instead, it was found that a strong correlation existed between plastic strain 
amplitude and distance to grain boundaries, with regions close to grain boundaries exhibiting both the highest levels of plastic strain and lowest levels of plastic strain (figure 1c).

To interpret these results a spectral based full-field crystal plasticity model [6] was employed. In this case, a synthetic starting polycrystal was constructed. The grains in this polycrystal were then populated with orientations taken from the experimentally measured texture. This 'polycrystal' was subjected to a simulated tensile test where the slip system level constitutive law parameters were adjusted in order to capture both the macroscopic stress-strain curve as well as the distribution of local plastic strain amplitudes. Twinning was not considered in the simulations, since the experiments showed (as expected) $<5$ area $\%$ twins at the highest level of strain. It was found that, in order to predict the experimental results, one needed to have a sufficiently high stress for activating non-basal (hard) slip systems compared to that required for basal systems (at least 10 times higher CRSS) [5]. The simulations also predicted the observed correlation between strain amplitude and distance to grain boundaries (figure 1c). From the results of the simulation it was possible to show that regions of high strain amplification occurred in grains deforming predominantly by basal slip that were adjacent to grains deforming predominantly by non-basal slip. This result shows the first order effect that neighborhood plays in determining the plastic response of such highly plastically anisotropic magnesium alloys [7].

\section{References:}

[1] J. Jain, W. J. Poole and C. W. Sinclair, Mater. Sci. Eng. A, 547 (2012) 128-137

[2] P. Tomlinson, et al., Metall. Mater. Trans. A, 44 (2013) 2970-2983

[3] R. A. Lebensohn, C. N. Tomé, Acta Mater., 41 (1993) 2611-2624

[4] G. Martin, C.W. Sinclair, J.H. Schmitt, Scripta Mater., 68(2013) 695-698

[5] G. Martin, C.W. Sinclair, R.A. Lebensohn, Mater. Sci. Eng. A, in press.

[6] R.A. Lebensohn, Acta Materialia 49 (2001) 2723-273

[7] This research was supported by funding from the NSERC Magnesium Strategic Research Network. More information on the Network can be found at www.MagNET.ubc.ca

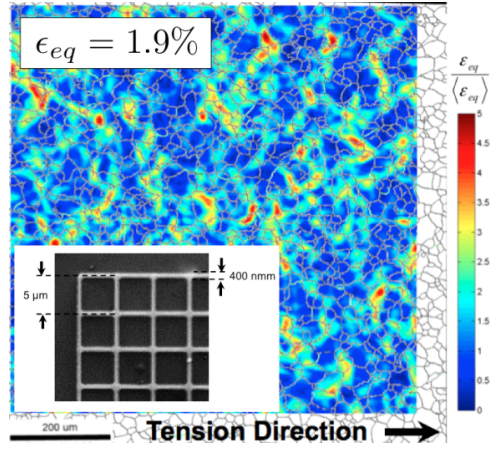

(a)

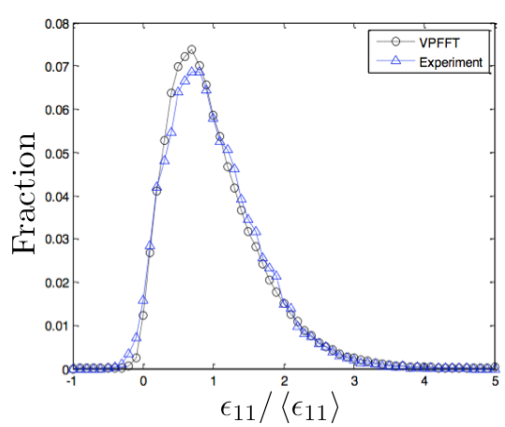

(b)

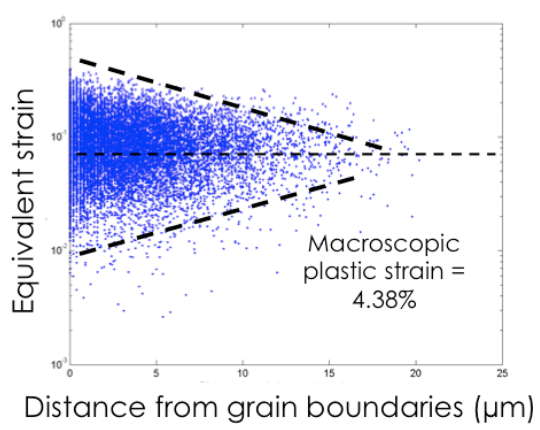

(c)

Figure 1. a) Detail of grid used for image correlation measurements superimposed on map of normalized Von Mises equivalent plastic strain b) plastic strain distribution measured experimentally and simulated using the VPFFT code c) experimental data from image correlation showing the correspondence between high strain amplitude and proximity to grain boundaries. 